\title{
Increasing symbol rate by symbol decision based on spatial luminance distribution for rolling-shutter optical camera communication
}

\author{
Taiki Tanemura ${ }^{1}$ and Wataru Chujo ${ }^{1,}$ a) \\ ${ }^{1}$ Department of Electrical and Electronic Engineering, Meijo University, \\ 1-501 Shiogamaguchi, Tempaku-ku, Nagoya 468-8502, Japan \\ a)wchujo@meijo-u.ac.jp
}

\begin{abstract}
In rolling-shutter (RS) optical camera communication (OCC), as the ratio of the exposure time to the symbol length increases, it becomes difficult to achieve error-free transmission due to inter-symbol interference. In this study, to increase the symbol rate, symbol decision based on spatial luminance distribution is adapted for RS OCC. When the symbol rate increases and the ratio is close to 1 , it is difficult to achieve error-free transmission by conventional symbol decision based on pixel value. However, even when the ratio increases up to 1, error-free transmission is achieved by the symbol decision based on spatial luminance distribution.
\end{abstract}

Keywords: visible light communication, optical camera communication, image sensor, rolling shutter, exposure time, spatial luminance distribution

Classification: Wireless Communication Technologies

\section{References}

[1] C. Danakis, M. Afgani, G. Povey, I. Underwood, and H. Haas, "Using a CMOS camera sensor for visible light communication," IEEE Globecom Workshops, Anaheim, USA, pp. 1244-1248, Dec. 2012. DOI: 10.1109/GLOCOMW. 2012.6477759

[2] H. Aoyama and M. Oshima, "Line scan sampling for visible light communication: theory and practice," IEEE International Conference on Communications, London, UK, pp. 5060-5065, June 2015. DOI: 10.1109/ICC.2015.7249126

[3] K. Ohshima, T. Naramoto, K. Yamaguchi, and W. Chujo, "Rolling-shutter-based asynchronous optical camera communication by a cycle pattern of received symbols using smartphones," IEICE Commun. Express, vol. 8, no. 3, pp. 49-54, March 2019. DOI: 10.1587/comex.2018XBL0141

[4] W. Chujo and M. Kinoshita, "Rolling-shutter-based 16QAM optical camera communication by spatial luminance distribution," IEICE Commun. Express, vol. 8, no. 12, pp. 566-571, Dec. 2019. DOI: 10.1587/comex.2019GCL0055 


\section{Introduction}

In rolling-shutter (RS) optical camera communication (OCC) between LED transmitter and RS camera receiver, exposure time, $T_{e x}$, of off-the-shelf camera is longer than exposure time interval, $T_{t}[1,2]$. As the symbol length, $T_{s}$, decreases and comes close to $T_{t}$, it becomes difficult to achieve error-free transmission owing to long exposure time.

However, in some smartphones' built-in cameras, error-free transmission has been achieved even when $T_{s} / T_{t}=1$ at IEICE Communications Express [3]. Although $T_{e x}$ of the built-in image sensor is unknown, error-free transmission has been achieved by adjusting the threshold pixel value, $p_{t h}$.

In this study, to increase the symbol rate, symbol error rate (SER) is measured while changing the ratio, $T_{e x} / T_{s}$. In general, 8-bit pixel value of the captured LED image is used for symbol decision. When the ratio is much less than 1, error-free transmission is expected with conventional symbol decision based on the pixel value. However, error-free transmission based on the pixel value is difficult when the ratio is close to 1. In order to achieve error-free transmission when the ratio increases up to 1 , symbol decision based on spatial luminance distribution [4] is adapted for RS OCC.

\section{Relationship between exposure time and symbol length}

SER is most likely determined by the ratio, $T_{e x} / T_{s}$. Figure 1 shows the effect of exposure time on received symbol pattern when repetition symbol of " 1 " and " 0 " is transmitted with on-off keying (OOK). In Figs. 1(a), (b), and (c), $T_{s} / T_{t}=1$ is assumed and the sensitivity of the image sensor is assumed to be high.

Figure 1(a) shows a schematic diagram illustrating the relationship between $T_{e x}$ and received pixel value when $T_{e x} / T_{s} \approx 0$, where $\Delta t$ is offset time between shutter timing of the camera and the symbol timing, and $p_{1}$ and $p_{0}$ is the received pixel value from 0 to 255 when symbol " 1 " and " 0 " is transmitted, respectively. When $T_{e x} / T_{s} \approx 0$, error-free transmission is expected at any shutter timing because $T_{e x}$ does not overlap between symbols " 1 " and " 0 ." Therefore, the pixel value, $p_{1}$ and $p_{0}$, is expected to be 255 and 0 , respectively.

On the other hand, Fig. 1(b) shows the diagram when $0 \ll T_{e x} / T_{s} \ll 1$. The exposure time, $T_{e x}$, overlaps between symbols " 1 " and " 0 ." The pixel value, $p_{1}$, is always expected to be 255 . On the other hand, the pixel value, $p_{0}$, is given by

$$
p_{o}=a\left(\Delta t+T_{e x}-T_{s}\right) \geq 0,
$$

where $a$ is an arbitrary constant determined with the sensitivity of the image sensor, light intensity of the transmitter, and so on. As $\Delta t$ and $T_{e x}$ increase, $p_{0}$ increases more. However, $p_{1}$ and $p_{0}$ have different pixel values when $0 \ll T_{e x} / T_{s} \ll 1$. Error-free transmission is expected by choosing $p_{t h}$ appropriately for symbol decision.

Moreover, Fig. 1(c) shows the diagram when $T_{e x} / T_{s} \approx 1$. The pixel value, $p_{0}$, increases further. Both $p_{1}$ and $p_{0}$ are expected to be 255 . It seems to be impossible to make a symbol decision based on the pixel value.

To clarify the effect of $T_{e x}$ on received symbol pattern, Fig. 1(d) shows examples of measured pixel values while changing the ratio, $T_{e x} / T_{s}$, where $T_{s} / T_{t}=3$. A 


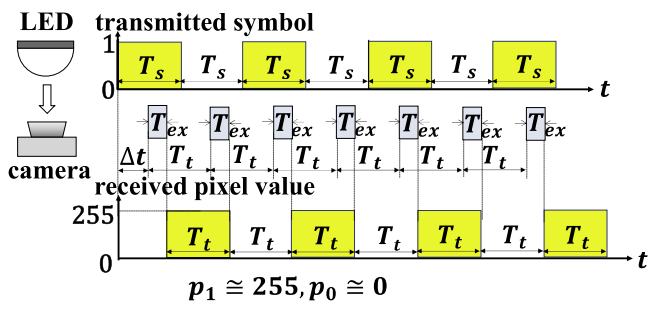

(a) $T_{e x} / T_{s} \approx 0,\left(T_{s} / T_{t}=1\right)$

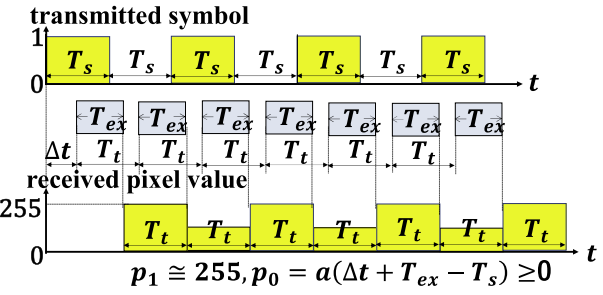

(b) $0 \ll T_{e x} / T_{s} \ll 1,\left(T_{s} / T_{t}=1\right)$
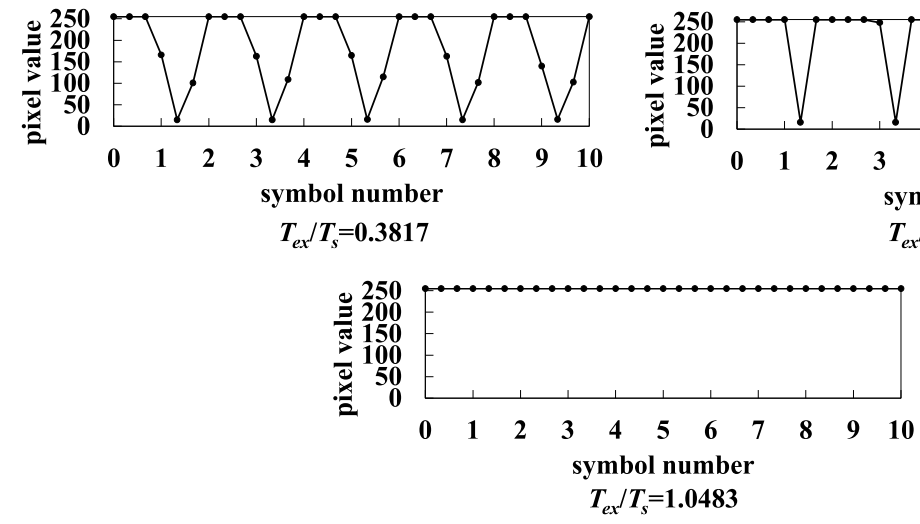

(d) examples of measured pixel values $\left(T_{s} / T_{t}=3\right)$

Fig. 1. Effect of exposure time, $T_{e x}$, on received symbol pattern while changing the ratio, $T_{e x} / T_{s}$, where $T_{s}$ is the symbol length, $T_{e x}$ is the exposure time, $T_{t}$ is the exposure time interval, $\Delta t$ is offset time between shutter timing of the camera and the symbol timing, and $p_{1}$ and $p_{0}$ is the received pixel value from 0 to 255 when symbol " 1 " and " 0 " is transmitted, respectively.

symbol consists of three pixels. When $T_{e x} / T_{s}=0.3817$, three pixels values, $p_{1}$, are always 255. Although three pixels values, $p_{0}$, gradually increase, average of the pixel values, $p_{0}$, is less than 128 . If $p_{t h} \geq 128$, error-free transmission is achieved.

When $T_{e x} / T_{s}=0.7150$, two pixels values, $p_{0}=255$. However, the value of the remaining one pixel, $p_{0}<255$. Although average of three pixels values, $p_{0}$, increases further, error-free transmission is possible by choosing $p_{t h}<255$.

In contrast, when $T_{e x} / T_{s}=1.0483$, both the pixel values, $p_{1}=p_{0}=255$ owing to long exposure time. There is no difference between $p_{1}$ and $p_{0}$. It is impossible to make a symbol decision based on the pixel value. 


\section{SER measured with symbol decision based on pixel value}

SER was measured with symbol decision based on the received pixel value. Fig. 2 shows relationship between measured SER and the ratio, $T_{e x} / T_{s}$. Fig. 2(a) shows technical parameters of LED transmitter and RS camera receiver. Repetition symbol of " 1 " and " 0 " is transmitted with OOK. The symbol length, $T_{s}$, is chosen to integer multiple of the exposure time interval, $T_{t}$. Relationship between SER and $T_{e x} / T_{s}$ is investigated at various symbol rate by changing the ratio, $T_{S} / T_{t}$.

\begin{tabular}{|c|c|}
\hline \multicolumn{2}{|c|}{ LED transmitter } \\
\hline maximum total flux & 3500 lumens \\
\hline surface area & $26 \times 26 \mathrm{~mm}$ square \\
\hline full width at half maximum & 140 degrees \\
\hline maximum luminance & approx. $1 \mathrm{Mcd} / \mathrm{m}^{2}$ \\
\hline FPGA board & $\mathrm{Zybo} \mathrm{Z7}-20$ \\
\hline
\end{tabular}

\begin{tabular}{|c|c|c|c|}
\hline \multicolumn{4}{|c|}{ RS camera receiver } \\
\hline lens focal length & $25 \mathrm{~mm}$ & \multirow{2}{*}{ exposure time } & \multirow{2}{*}{$\begin{array}{c}\text { lowest: } 15.97 \mu \mathrm{s} \\
\text { interval: } 12.38 \mu \mathrm{s}\end{array}$} \\
\hline f-number & 1.2 & & \\
\hline field of view & 16.7 degrees & \multirow{2}{*}{ image size } & \multirow{2}{*}{$\begin{array}{c}\text { VGA } \\
(640 \times 480 \text { pixels })\end{array}$} \\
\hline image sensor & IMX219 & & \\
\hline line interval/ line rate & $12.38 \mu \mathrm{sec} / 80.78 \mathrm{k}$ lines $/ \mathrm{s}$ & FPGA board & Ultra96 \\
\hline
\end{tabular}

(a) technical parameters of LED transmitter and RS camera receiver

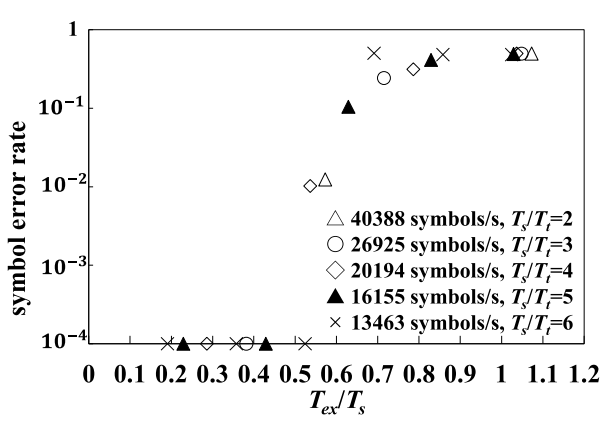

(b) $p_{t h}=160$

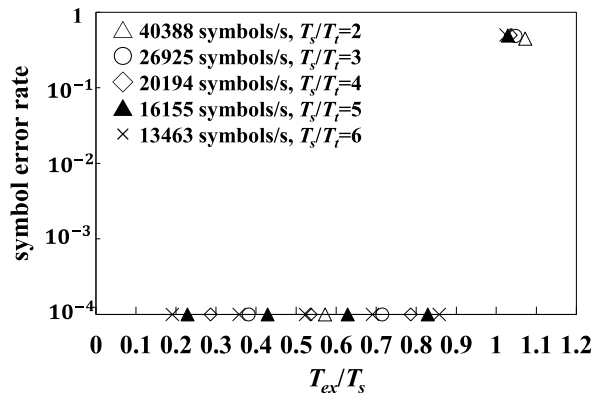

(c) $p_{t h}=254$

Fig. 2. SER measured with symbol decision based on pixel value.

Figure 2(b) shows relationship between SER and $T_{e x} / T_{s}$ when $p_{t h}=160$, where SER $=10^{-4}$ indicates error-free transmission. Since $p_{t h}$ is not high, errors may occur as $T_{e x} / T_{s}$ increases. Actually, errors occur when $T_{e x} / T_{s}$ is more than 0.5363. This is due to increase of the pixel value, $p_{0}$. When $0 \ll T_{e x} / T_{s} \ll 1$, it is obvious that long exposure time degrades SER.

In contrast, Fig. 2(c) shows SER and $T_{e x} / T_{s}$ when $p_{t h}=254$. Since $p_{t h}$ is high, error-free transmission is achieved up to $T_{e x} / T_{s}=0.858$. The pixel value, $p_{0}$, increases as $T_{e x} / T_{s}$ increases. However, if $p_{0}<255$, it is possible to make a symbol decision by increasing $p_{t h}$ up to the maximum value, 255. Possibility of the symbol decision depends on the sensitivity of the image sensor. We considered the reason why error-free transmission was achieved using smartphones' built-in RS cameras even when $T_{s} / T_{t}=1$ at IEICE Communications Express [3]. The reason may be that 
the sensitivity of the image sensor was not high. On the other hand, in this study, the sensitivity of the image sensor is too high to achieve error-free transmission when $T_{e x} / T_{s}=1$ with symbol decision based on the pixel value.

\section{SER measured with symbol decision based on spatial luminance distribution}

When $T_{e x} / T_{s}$ increases up to $1, p_{0}$ is saturated to 255 and whiteout occurs in the captured image. It is impossible to make a symbol decision based on the pixel value. However, even when $p_{0}$ is saturated on the LED surface image, spatial luminance distribution of the surface gradually decreases towards end part of the surface. Therefore, spatial luminance distribution is effective for symbol decision [4].

RS camera captures the spatial luminance distribution of LED surface at each time interval. In the captured image, white pixels indicate symbol "1" after adjusting $p_{t h}$. Although $p_{0}$ is saturated to 255 on the surface, number of white pixels varies on each interval in accordance with OOK modulation.

Figure 3(a) shows an example of captured LED image when repetition symbol of " 1 " and "0" is transmitted with OOK, where $T_{s} / T_{t}=6$ and $T_{e x} / T_{s}=1.0242$.

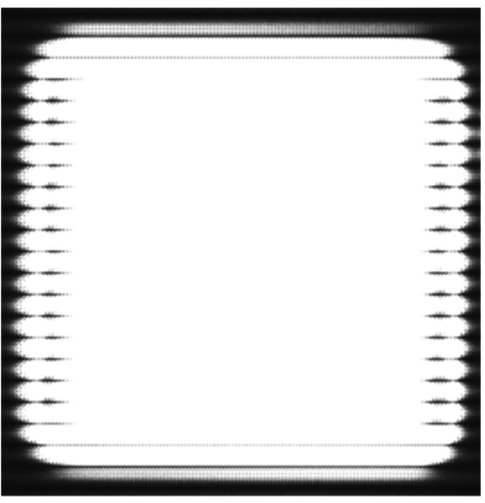

(a) an example of captured LED image

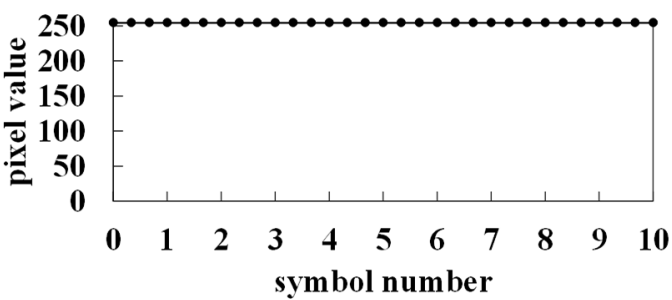

(b) pixel values on the middle part of the image

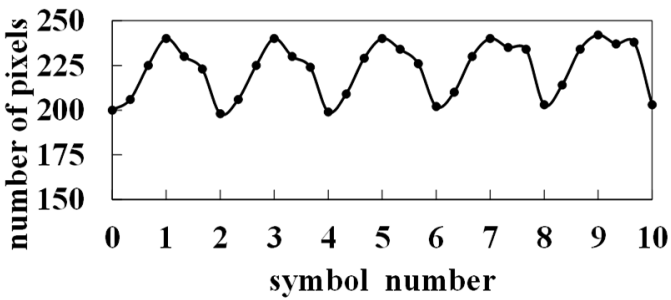

(c) number of white pixels on the image $\left(p_{t h}=254\right)$

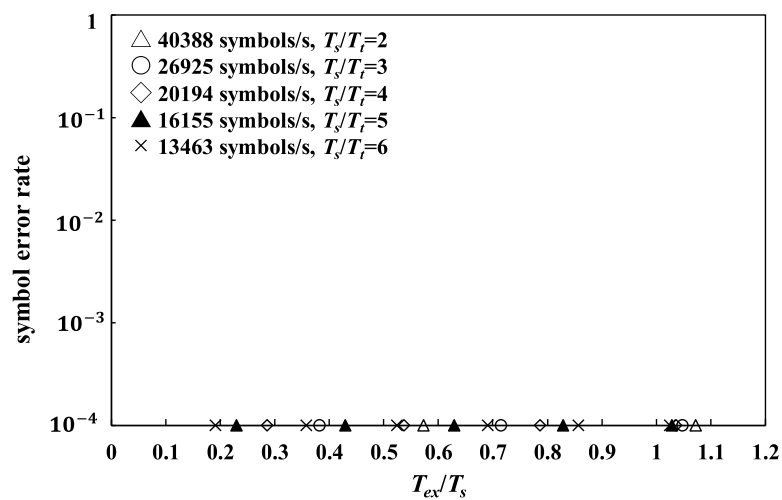

(d) relationship between measured SER and the ratio, $T_{e x} / T_{s}\left(p_{t h}=254\right)$

ㅇ IEICE 2020

DOI: $10.1587 /$ comex.2020COL0023 Received June 29, 2020 Accepted July 27, 2020

Publicized August 6, 2020

Copyedited December 1, 2020 
Each pixel line of the camera is sequentially exposed from top to bottom. Since $p_{0}$ is saturated on the LED surface image, whiteout occurs at the middle part of the image. It is impossible to make a symbol decision with the pixel value. However, the spatial luminance distribution of LED surface gradually decreases towards end part of the surface. Pixel values, $p_{1}$ and $p_{0}$, on right- and left-edge parts of the image vary in accordance with OOK. Therefore, after adjusting $p_{t h}$, number of white pixels varies in accordance with OOK.

Figure 3(b) shows measured pixel values on the middle part of the captured image, where $T_{s} / T_{t}=3$ and $T_{e x} / T_{s}=1.0483$. Symbol length is three times as long as the exposure time interval. Since all the pixel values are saturated at the maximum value, it is impossible to make a symbol decision. On the other hand, Fig. 3(c) shows number of white pixels on each interval after adjusting $p_{t h}=254$. It is possible to make a symbol decision by averaging the number of white pixels on symbol " 1 " and " 0 " every 3 pixels.

Figure 3(d) shows relationship between measured SER and the ratio, $T_{e x} / T_{s}$, with symbol decision based on the spatial luminance distribution, where $p_{t h}=254$. Error-free transmission is achieved up to $T_{e x} / T_{s}=1.0725$. When the ratio of the symbol length to the exposure time interval, $T_{s} / T_{t}=2$, symbol rate increases up to 40.388 kilo-symbols per second (symbols/s). Even when $T_{e x} / T_{s} \approx 1$, error-free transmission is achieved.

\section{Conclusion}

In this study, to increase the symbol rate for RS OCC, the symbol decision based on spatial luminance distribution was adapted for RS OCC with OOK. When the ratio of the exposure time to the symbol length, $T_{e x} / T_{s} \approx 0$, error-free transmission was achieved regardless of the threshold pixel value, $p_{t h}$, with conventional symbol decision based on pixel value.

On the other hand, when $0 \ll T_{e x} / T_{s} \ll 1$, error-free transmission was achieved by carefully choosing $p_{t h}$ with the symbol decision based on pixel value. However, error-free transmission depends on the sensitivity of the image sensor.

To increase the symbol rate further, when $T_{e x} / T_{s} \approx 1$, error-free transmission was achieved with the symbol decision based on spatial luminance distribution. Even though pixel values, $p_{1}$ and $p_{0}$, on the middle part of LED image are saturated and whiteout occurs, $p_{1}$ and $p_{0}$ on the edge part of the image help to make a symbol decision. Error-free transmission was achieved up to $T_{e x} / T_{S}=1.0725$ with the symbol decision based on spatial luminance distribution. Symbol rate increased up to 40.388 kilo-symbols/s. 